\title{
Europace
}

\section{Diagnosis, family screening and treatment of inherited arrhythmogenic diseases in Europe: results of the European Heart Rhythm Association survey --Manuscript Draft--}

\begin{tabular}{|c|c|}
\hline Manuscript Number: & EUPC-D-20-00865 \\
\hline Full Title: & $\begin{array}{l}\text { Diagnosis, family screening and treatment of inherited arrhythmogenic diseases in } \\
\text { Europe: results of the European Heart Rhythm Association survey }\end{array}$ \\
\hline Article Type: & EHRA Survey \\
\hline Keywords: & $\begin{array}{l}\text { sudden cardiac death; sudden cardiac arrest; inherited arrhythmogenic diseases; } \\
\text { inherited primary arrhythmia syndromes; cardiomyopathies; genetic heart disease }\end{array}$ \\
\hline Corresponding Author: & $\begin{array}{l}\text { Giulio Conte, M.D., Ph.D. } \\
\text { Cardiocentro Ticino } \\
\text { Lugano, SWITZERLAND }\end{array}$ \\
\hline \multicolumn{2}{|l|}{$\begin{array}{l}\text { Corresponding Author Secondary } \\
\text { Information: }\end{array}$} \\
\hline Corresponding Author's Institution: & Cardiocentro Ticino \\
\hline \multicolumn{2}{|l|}{$\begin{array}{l}\text { Corresponding Author's Secondary } \\
\text { Institution: }\end{array}$} \\
\hline First Author: & Giulio Conte, M.D., Ph.D. \\
\hline \multicolumn{2}{|l|}{ First Author Secondary Information: } \\
\hline \multirow[t]{9}{*}{ Order of Authors: } & Giulio Conte, M.D., Ph.D. \\
\hline & Daniel Scherr, MD \\
\hline & Radoslaw Lenarczyk, MD \\
\hline & Estelle Gandjbachkh, MD \\
\hline & Stéphane Boulé, MD \\
\hline & Michael D. Spartalis, MD \\
\hline & Elijah R. Behr, MD \\
\hline & Arthur Wilde, MD \\
\hline & Tatjana Potpara, MD, PhD \\
\hline \multicolumn{2}{|c|}{ Order of Authors Secondary Information: } \\
\hline Abstract: & $\begin{array}{l}\text { The spectrum of inherited arrhythmogenic diseases (IADs) includes disorders without } \\
\text { overt structural abnormalities (i.e. primary inherited arrhythmia syndromes) and } \\
\text { structural heart diseases (i.e. arrhythmogenic ventricular cardiomyopathy, hypertrophic } \\
\text { cardiomyopathy). The aim of this European Heart Rhythm Association (EHRA) survey } \\
\text { was to evaluate current clinical practice and adherence to } 2015 \text { European Society of } \\
\text { Cardiology Guidelines regarding the management of patients with IADs. } \\
\text { A } 24 \text {-item centre-based online questionnaire was presented to the EHRA Research } \\
\text { Network Centres and the European Cardiac Arrhythmia Genetics Focus Group } \\
\text { members. There were } 46 \text { responses from } 20 \text { different countries. The survey revealed } \\
\text { that } 37 \% \text { of centres did not have any dedicated unit focusing on patients with IADs. } \\
\text { Provocative drug challenges were widely used to rule-out Brugada syndrome (BrS) } \\
\text { ( } 91 \% \text { of centres), while they were used in a minority of centres during the diagnostic } \\
\text { assessment of long QT syndrome (11\%), early repolarization syndrome (12\%) or } \\
\text { catecholaminergic polymorphic ventricular tachycardia (18\%). While all centres } \\
\text { advised family clinical screening with ECGs for all first-degree family members of } \\
\text { patients with IADs, genetic testing was advised in family members of probands with } \\
\text { positive genetic testing by } 33 \% \text { of centres. SCD risk stratification was straightforward } \\
\text { and in line with current guidelines for hypertrophic cardiomyopathy, while it was } \\
\text { controversial for other diseases (i.e. BrS). Finally, indications for ventricular mapping }\end{array}$ \\
\hline
\end{tabular}


and ablation procedures in $\mathrm{BrS}$ were variable and not in agreement with current guidelines in up to $54 \%$ of centres.

Powered by Editorial Manager ${ }^{\circledR}$ and ProduXion Manager ${ }^{\circledR}$ from Aries Systems Corporation 


\begin{abstract}
The spectrum of inherited arrhythmogenic diseases (IADs) includes disorders without overt structural abnormalities (i.e. primary inherited arrhythmia syndromes) and structural heart diseases (i.e. arrhythmogenic ventricular cardiomyopathy, hypertrophic cardiomyopathy). The aim of this European Heart Rhythm Association (EHRA) survey was to evaluate current clinical practice and adherence to 2015 European Society of Cardiology Guidelines regarding the management of patients with IADs.
\end{abstract}

A 24-item centre-based online questionnaire was presented to the EHRA Research Network Centres and the European Cardiac Arrhythmia Genetics Focus Group members. There were 46 responses from 20 different countries. The survey revealed that $37 \%$ of centres did not have any dedicated unit focusing on patients with IADs. Provocative drug challenges were widely used to rule-out Brugada syndrome $(\mathrm{BrS})$ (91\% of centres), while they were used in a minority of centres during the diagnostic assessment of long QT syndrome (11\%), early repolarization syndrome $(12 \%)$ or catecholaminergic polymorphic ventricular tachycardia (18\%). While all centres advised family clinical screening with ECGs for all first-degree family members of patients with IADs, genetic testing was advised in family members of probands with positive genetic testing by $33 \%$ of centres. SCD risk stratification was straightforward and in line with current guidelines for hypertrophic cardiomyopathy, while it was controversial for other diseases (i.e. BrS). Finally, indications for ventricular mapping and ablation procedures in $\mathrm{BrS}$ were variable and not in agreement with current guidelines in up to $54 \%$ of centres.

Key words: sudden cardiac death; sudden cardiac arrest; inherited arrhythmogenic diseases; inherited primary arrhythmia syndromes; cardiomyopathies; genetic heart disease. 


\title{
Diagnosis, family screening and treatment of inherited arrhythmogenic diseases in Europe: results of the European Heart Rhythm Association survey
}

\author{
Giulio Conte MD PhD ${ }^{1,2,3}$, Daniel Scherr MD ${ }^{4}$, Radoslaw Lenarczyk MD ${ }^{5}$, Estelle \\ Gandjbachkh MD ${ }^{6}$, Stéphane Boulé MD ${ }^{7}$, Michael D. Spartalis MD ${ }^{8}$, Elijah R. Behr MD \\ Arthur Wilde $\mathrm{MD}^{10}$ and Tatjana Potpara MD $\mathrm{PhD}^{11}$
}

(1) Division of Cardiology, Cardiocentro Ticino, Lugano, Switzerland;

(2) Faculty of Biomedical Sciences, USI, Lugano, Switzerland;

(3) Centre for Computational Medicine in Cardiology, Faculty of Informatics, Università della

Svizzera Italiana, Lugano, Switzerland;

(4) Division of Cardiology, Medical University of Graz, Austria;

(5) First Department of Cardiology and Angiology, Silesian Centre for Heart Disease, Zabrze, Poland;

(6) Sorbonne Universités, APHP, Cardiology Institute, ICAN, Pitié-Salpêtrière University Hospital, Paris, France;

(7) Department of Cardiology, Hôpital privé Le Bois, Lille, France;

(8) Division of Cardiology, Onassis Cardiac Surgery Center, Athens, Greece;

(9) Cardiology Clinical Academic Group, St. George's, University of London and St.

George's University Hospitals NHS Foundation Trust, London, UK;

(10) (ERN GUARDHEART);

(11) School of Medicine, University of Belgrade, Belgrade, Serbia; cardiology Clinic, Clinical Centre of Serbia, Belgrade, Serbia

Word count: 2303

\section{Corresponding author}

Giulio Conte MD, PhD

Cardiocentro Ticino

Lugano, Switzerland

Mail: giulio.conte@cardiocentro.org

Tel. +41918053350

Fax +41918053173 


\begin{abstract}
The spectrum of inherited arrhythmogenic diseases (IADs) includes disorders without overt structural abnormalities (i.e. primary inherited arrhythmia syndromes) and structural heart diseases (i.e. arrhythmogenic ventricular cardiomyopathy, hypertrophic cardiomyopathy). The aim of this European Heart Rhythm Association (EHRA) survey was to evaluate current clinical practice and adherence to 2015 European Society of Cardiology Guidelines regarding the management of patients with IADs.

A 24-item centre-based online questionnaire was presented to the EHRA Research Network Centres and the European Cardiac Arrhythmia Genetics Focus Group members. There were 46 responses from 20 different countries. The survey revealed that $37 \%$ of centres did not have any dedicated unit focusing on patients with IADs. Provocative drug challenges were widely used to rule-out Brugada syndrome $(\mathrm{BrS})$ (91\% of centres), while they were used in a minority of centres during the diagnostic assessment of long QT syndrome (11\%), early repolarization syndrome $(12 \%)$ or catecholaminergic polymorphic ventricular tachycardia (18\%). While all centres advised family clinical screening with ECGs for all first-degree family members of patients with IADs, genetic testing was advised in family members of probands with positive genetic testing by $33 \%$ of centres. SCD risk stratification was straightforward and in line with current guidelines for hypertrophic cardiomyopathy, while it was controversial for other diseases (i.e. BrS). Finally, indications for ventricular mapping and ablation procedures in $\mathrm{BrS}$ were variable and not in agreement with current guidelines in up to $54 \%$ of centres.
\end{abstract}

Key words: sudden cardiac death; sudden cardiac arrest; inherited arrhythmogenic diseases; inherited primary arrhythmia syndromes; cardiomyopathies; genetic heart disease. 


\section{Introduction}

The inherited arrhythmogenic diseases (IADs) are a heterogeneous group of genetic heart diseases predisposing to sudden cardiac arrest (SCA), including primary inherited arrhythmia syndromes (IAS) and cardiomyopathies (CMPs). ${ }^{1,2}$

The diagnostic approach to IADs is heterogeneous across European centres with regard to the use of provocative drug challenges, genetic testing, and family screening of relatives. ${ }^{3}$ Risk prediction of sudden cardiac death (SCD) and indication to primary prevention using implantable cardioverter-defibrillator (ICD) still represent important unmet clinical needs for most of IADs. ${ }^{3}$

In 2013 and 2015 European Heart Rhythm Association (EHRA) and the European Society of Cardiology (ESC), together with its associated partners around the world defined specific recommendations on the diagnosis and treatment of IAS. ${ }^{1,2}$ In the following years new therapeutic options have become more widely available, e.g. substrate ablation in BrS, which were only touched upon in the aforementioned documents. ${ }^{4,5}$

The aim of this EHRA survey was to evaluate contemporary clinical practice in the management of patients with IADs, and adherence to the current 2015 ESC Guidelines for the management of patients with ventricular arrhythmias and the prevention of SCD. ${ }^{2}$ The previous EHRA survey on IADs was conducted in $2014 .^{3}$

\section{Methods and results}

The EHRA Scientific Initiatives Committee conducted the present survey in collaboration with the European Cardiac Arrhythmia Genetics' Focus Group (ECGen) and the European Reference Network for Rare and Low Prevalence Complex Diseases of the Heart (ERN GUARD-Heart). A centre-based on-line questionnaire was constructed to collect information about current diagnostic and therapeutic management of patients with IADs in Europe, 
including the following diseases: Brugada syndrome (BrS), long-QT syndrome (LQTS), short-QT syndrome (SQTS), catecholaminergic polymorphic ventricular tachycardia (CPVT), idiopathic ventricular fibrillation (IVF), early repolarization syndrome (ERS), progressive cardiac conduction defect (PCCD), arrhythmogenic cardiomyopathy (ACM) and hypertrophic cardiomyopathy (HCM). The link was sent out to the EHRA Research Network Centres and ECGen members. Of 54 centres answering the general questions about centre type, volume and specialty, 46 centres $(85.2 \%)$ completed the questionnaire and are included in this analysis. Hence, the results are reported as number and percentage of 46 , unless otherwise specified (in case of missing response to a specific question, the total number of replies collected for that question is provided in the respective figure legend).

The 46 respondents represented centres from the following 20 countries: Austria, Belgium, Czech Republic, Denmark, France, Georgia, Germany, Greece, Italy, Malta, The Netherlands, Poland, Portugal, Russian Federation, Serbia, Slovenia, Spain, Switzerland, Turkey, and Uzbekistan. Among participating centres, 70\% were university hospitals, 17\% non-university public hospitals, and $13 \%$ private hospitals/practice. Of the respondents, $85 \%$ had specific competences in cardiac electrophysiology, $15 \%$ in cardio-genetics, $6 \%$ in cardiac imaging and $4 \%$ in paediatric cardiology or congenital heart diseases.

Half of all respondents declared the presence of dedicated units focusing on patients with IADs at their centre, while there were no specific IADs units in $37 \%$ of centres. In $13 \%$ of centres, there was a single dedicated unit for patients with IAS or CMPs. (Figure 1)

Figure 1. Presence of IADs units in the EHRA Research network and ECGen member centres. 
Out of 46 centres, $14(30 \%)$ managed $>100$ patients with IADs annually, $10(22 \%)$ and 12 (26\%) centres managed 50-100 patients with IAS and CMPs, respectively, and 25 (54\%) and $24(52 \%)$ centres managed $<50$ patients with IAS and CMPs, respectively. Moreover, there were 29 centres $(63 \%)$ managing paediatric patients $(<16$ years) with IAS and CMPs, and 1 and 2 centres managing exclusively paediatric patients with IAS and CMPS, respectively.

\section{Diagnostic assessment}

All responding centres reported the use of baseline 12-lead electrocardiogram (ECG) as an important tool in the initial diagnostic evaluation of all suspected IAD cases. In case of a nondiagnostic baseline ECG, the most common indication to perform a pharmacological challenge was the presence of ECG abnormalities suggestive of IAS (e.g., Brugada type 2 ECG, malignant ER pattern, borderline QTc, atrioventricular [AV] conduction disturbances) with or without syncopal or aborted SCA $(80 \%)$, followed by any aborted SCA of unknown aetiology (65\%). Other indications for drug challenge included family history of SCD, family screening for IAS, AF in otherwise healthy subjects $<50$ years of age, sinus node dysfunction 
(SND) in patients $<50$ years of age, and any syncopal episode of cardiac origin in patients $<50$ years of age. (Figure 2)

Figure 2. Indications for pharmacological challenge.

Provocative drug challenges were widely used to rule-out $\mathrm{BrS}$ (91\% of centres), while they were used in a minority of centres for the diagnostic assessment of LQTS (11\%), ERS (12\%) or CPVT $(18 \%)$.

Drug challenge was performed in a cardiac catheterization laboratory in $44 \%$ of centres, in cardiac intensive care unit in $24 \%$, in the cardiology ward in $24 \%$, and in outpatient clinic in the remaining $8 \%$ of centres. Ajmaline was the most commonly used drug to rule-out $\mathrm{BrS}$ ( $88 \%$ of centres), followed by flecainide $(5 \%)$ or other drugs (i.e., propafenone, procainamide, 7\%). A signal-averaged ECG was performed in $\mathrm{BrS}$ patients in $9 \%$ of centres, and in $\mathrm{ACM}$ patients in $37 \%$ of centres. The rate of diagnostic examinations performed in the assessment of different IAS and CMPs is shown in Figure 3. 
The proportion of centres with and without dedicated IAS units undertaking genetic testing, according to the specific IAS or CM, is shown in Figure 4. Notably, centres with dedicated IAS units had a higher rate of genetic testing compared with centres without dedicated IAS units.

Figure 3. Rate of diagnostic exams in patients with IADs.

Total answers: BrS 46; CPVT 44; LQT 46; IVF 43; ACM 44; HCM 46.

BrS Brugada syndrome; CPVT Catecholaminergic polymorphic ventricular tachycardia; LQT long-QT syndrome; IVF idiopathic ventricular fibrillation; ACM Arrhythmogenic cardiomyopathy; HCM hypertrophic cardiomyopathy; CMR cardiac magnetic resonance. 
Figure 4. Rate of genetic testing in centres with and without dedicated IAS units.

Total answers: 46 (29 centres with dedicated IAS units and 17 centres with no dedicated IAS units)

BrS Brugada syndrome; CPVT Catecholaminergic polymorphic ventricular tachycardia; LQTS long-QT syndrome; IVF idiopathic ventricular fibrillation; ACM Arrhythmogenic cardiomyopathy; HCM hypertrophic cardiomyopathy

\section{SCD risk stratification}

The majority of centres $(>73 \%)$ considered baseline 12-lead ECG findings as prognostic indicators in IADs, particularly for BrS, LQT and ERS.

Electrophysiology study with programmed ventricular stimulation (PVS) was considered a tool for SCD risk stratification by $63 \%$ of centres for BrS, $33 \%$ for IVF, $24 \%$ for PCCD, $46 \%$ for ACM, and $17 \%$ for HCM. Only a minority of centres (10\%) uses PVS for risk stratification purposes in patients with other IADs (CPVT, SQTS, ERS). Specific indications to PVS in BrS are shown in Figure 5. 
Figure 5. Rate of specific indications to programmed ventricular stimulation in patients Brugada syndrome.

Total answers: 40

SCD sudden cardiac death.

The majority of centres (96\%) used a PVS protocol with 2 ventricular sites and up to 3 extrastimuli, while only $4 \%$ of centres less than 3 extrastimuli. Cardiac substrate invasive mapping data were used for SCD risk stratification in $5 \%$ of centres for $\mathrm{BrS}, 11 \%$ for IVF, and $15 \%$ for ACM. Genetic testing results were used for SCD risk stratification in $65 \%$ of centres for LQTS, $24 \%$ for BrS, 35\% for CPVT, $28 \%$ for IVF, $16 \%$ for PCCD, $5 \%$ for ERS, $37 \%$ for ACM, and $46 \%$ for HCM. CMR was considered a risk stratification tool by $78 \%$ of centres for ACM, and 73\% for HCM. Importantly, all centres declared the use of the HCM Risk-SCD calculator to estimate the risk of SCD at 5 years in all patients with HCM.

\section{Family screening}

All centres advised family clinical screening with ECGs of all first-degree family members of patients with IADs. Genetic testing was advised in first-degree relatives of proband with a positive genetic testing by $33 \%$ of centres and only in symptomatic first-degree relatives by $2 \%$ of centres. 
Sodium channel blocker challenge in paediatric BrS family members was considered by $67 \%$ while $33 \%$ of centres do not perform drug challenges in paediatric subjects due to concerns over potential pro-arrhythmic drug effects (Figure 6). Moreover, most centres (90\%) recommended performing an ECG recording during fever in children with BrS or a family history of BrS.

Figure 6. Drug challenge in paediatric BrS family members.

Total answers: 27

SND sinus node dysfunction.

\section{Therapeutic management}

In patients with IADs, $20 \%$ and $5 \%$ of centres considered implanting single-chamber and dual-chamber ICD, respectively. S-ICD was considered as a therapeutic option by $29 \%$ of centres, if the sensing screening was appropriate and no anti-tachycardia pacing was expected. However, most centres (46\%) determined the type of implantable electronic devices (transvenous vs. subcutaneous and single vs. dual-chamber) according to the specific disease and patient's choice. The disease most commonly treated with S-ICD implantation, upon appropriate sensing screening, was IVF (73\%), followed by $\operatorname{BrS}(68 \%)$, and LQTS/ACM/HCM (54\%/51\%/44\%). A minority of centres (17\%) would not consider S-ICD 
implantation in IADs patients owing to concerns over the potential dynamic ECG changes and subsequent T-wave oversensing episodes.

Most centres (64\%) would utilize ventricular mapping and ablation procedures of arrhythmogenic substrate in $\mathrm{BrS}$ patients in the following conditions: ventricular arrhythmias recurrences/electrical storm in case of drug therapy failure (47\%); ventricular arrhythmias recurrences/electrical storm as first choice (36\%); and, high-risk features (symptoms/ECG/ventricular arrhythmias inducibility at PVS) without ventricular arrhythmias recurrences/electrical storm (17\%). The preferred procedural strategy adopted by most centres was endo-/epicardial mapping/ablation as the first approach for all cases (42\%), followed by epicardial only mapping/ablation as the first approach (35\%) and then endocardial only mapping/ablation as the first approach (19\%). With regards to pharmacological therapy with quinidine, $72 \%$ of centres reported availability of the drug in their country. In IADs patients with recurrent $\mathrm{AF}$, an ablation procedure, instead of pharmacological rhythm control therapy, was preferred by most of centres (93\%). The preferred ablation strategy was PVI in $73 \%$ of centres, followed by PVI plus ablation of non-PV triggers (27\%).

\section{Competitive athletes with IADs and no ICDs}

Most centres (96.2\%) advised competitive sport avoidance in IADs patients without ICDs, most commonly for patients with CPVT (93\%), ACM/HCM (88\%), and LQT-1 (71\%). Up to $50 \%$ of centres would advise sport avoidance also in competitive athletes with LQT with unknown genotype, BrS, or ERS.

\section{Discussion}

The findings of this survey highlighted several features of the current management of IADs in participating European centres: 1) one in three centres had no dedicated unit for the 
management of patients with IADs; 2) there was a considerable heterogeneity in indications for pharmacological challenge, genetic testing, family screening and sport avoidance, 3) genetic testing is more often performed in centres with dedicated IAS units; 4) SCD risk stratification was in line with current guidelines for some (i.e. HCM), but still heterogeneous for other IADs (i.e. BrS), and 5) the indications for ventricular mapping and ablation procedures in $\mathrm{BrS}$ were variable and guideline non-adherent in $54 \%$ of centres.

Current guidelines do not address specific indications for drug challenges for many IAS. $^{2}$ In our survey, provocative drug challenges were widely used to rule-out $\mathrm{BrS}$ (91\% of centres), while they were used in a minority of centres (11-18\%) for the diagnostic assessment of LQTS, CPVT, or ERS. Of note, despite the possibility that atrial arrhythmias and SND might be the first manifestation of an inherited electrical disorder especially in the young, only a few centres $(15 \%)$ would perform a drug test to rule-out BrS. ${ }^{6,7}$ This may be due to concerns about the specificity of drug challenges that have arisen since the ESC guidelines were published (e,g., ajmaline challenge for $\mathrm{BrS}$ can have yields of almost $30 \%$ in patients with AVNRT). ${ }^{8}$

As IVF is still considered a diagnosis of exclusion, an extensive diagnostic assessment should be performed in all unexplained SCA survivors to rule-out structural abnormalities or underlying IADs. ${ }^{1}$ In our survey, CMR was used in the evaluation of unexplained SCA survivors by only $67 \%$ of centres. Interestingly, $26 \%$ and $51 \%$ of centres did not perform exercise stress testing and/or provocative drug challenge, respectively, to rule out CPVT, BrS and LQTS in unexplained SCA survivors. It has been reported that up to $30 \%$ of the victims of swimming-related drowning had a cardiac ion channel mutation associated with LQT1 and CPVT; and that mutations associated with CPVT1, and to a lesser extent LQT1, are the most significant findings following post-mortem genetic testing in autopsy negative cases of SCD. ${ }^{9,10}$ 
As these conditions are usually associated with catecholaminergic triggers of arrhythmic events, absence of exercise or provocative testing from the investigative protocol may lead to misdiagnosis in a proportion of SCA survivors.

In the 2013 HRS/EHRA/APHRS expert consensus statement on the diagnosis and management of IAS, genetic testing was recommended for family members of a successfully genotyped proband. ${ }^{1}$ Interestingly, in our survey it was advised in first-degree relatives of proband with a positive genetic testing by only $33 \%$ of centres. Notably, genetic testing was more commonly performed in centres with dedicated IAS units. With regard to family screening in $\mathrm{BrS}$ patients, one out of three centres did not perform sodium channel blocker challenge in paediatric BrS family members, owing to the concern over potential proarrhythmic drug effects, which is in line with published evidence. ${ }^{6}$ For those centres performing drug challenge in children, the most common indication was the presence of symptoms, SND, atrial arrhythmias or the family's request. The ideal age for screening of BrS family members is unknown, and specific recommendations on screening are lacking, whilst limited supportive evidence suggests that postponing the screening until adult age is reasonable. ${ }^{11}$ Indeed, children with $\mathrm{BrS}$ represent a category of patients at a higher risk of lifethreatening arrhythmias during drug challenge and can have an age-dependent response to ajmaline. ${ }^{6,12}$ This important issue should be specifically addressed in the future guidelines.

Current guidelines state that ICD implantation may be considered in patients with a diagnosis of BrS who develop VF during PVS with two or three extrastimuli at two sites (IIb/C recommendation). ${ }^{2}$ Despite apparently limited utility of PVS in a pooled analysis of studies utilising PVS in BrS patients, PVS was used more often in BrS patients when compared with the previous 2014 EHRA-SIC survey (39\% vs. 63\%). ${ }^{3,13}$ Moreover, in the present survey, only a minority of centres (4\%) used a less aggressive PVS protocol with $<3$ extrastimuli. 
Surprisingly, a minority of centres reported using EPS for SCD risk stratification in CPVT or LQTS despite the lack of any supportive guidelines or data for this approach.

According to guidelines, catheter ablation may be considered in patients with $\mathrm{BrS}$ and history of electrical storms or repeated appropriate ICD shocks (IIb/C). ${ }^{2}$ In the 2014 EHRA-SIC survey, only $4 \%$ of centres declared ventricular ablation procedures, together with ICD implantation, as first-line therapy. In the present survey, $17 \%$ of centres would consider ablation procedures in high-risk BrS patients even in the absence of any documented spontaneous ventricular arrhythmias. In patients with recurrent arrhythmias, the proportion of centres regarding this as an indication for ablation procedures increased significantly over the last 6 years (from $8 \%$ to $56 \%$ ). The more recent evidence for substrate ablation in BrS as well as the need for a randomised case-control study will likely be addressed in future guidelines. ${ }^{4,5,13}$ Finally, although guidelines recommend competitive sport avoidance in patients with HCM, ACM and CPVT, 7-12\% of centres would not advise their patients accordingly. $^{2}$

\section{Conclusions}

Our survey suggests that the management of patients with IADs is heterogeneous across European centres with suboptimal adherence to current guidelines. Specific indications for drug challenges, genetic testing, ablation procedures, and family screening strategies are still unmet needs. This heterogeneity may reflect, in part, the absence of a dedicated unit for managing IAS patients. Further efforts to improve management of IADs across European countries are warranted. 


\section{Acknowledgements}

The production of this document is under the responsibility of the Scientific Initiatives Committee of the European Heart Rhythm Association: Tatjana S. Potpara (Chair), Radoslaw Lenarczyk (Co-Chair), Giulio Conte, Gheorghe Andrei Dan, Michal M. Farkowski, Malcolm Finlay, Estelle Gandjbakhch, Konstantinos E. Iliodromitis, Kristine Jubele, Deirdre A. Lane, Eloi Marijon, Francisco Marin, Frits Prinzen, and Daniel Scherr. This work was also conducted in collaboration with the European Cardiac Arrhythmia Genetics' Focus Group (ECGen) and the European Reference Network for Rare and Low Prevalence Complex Diseases of the Heart (ERN GUARD-Heart). 


\section{REFERENCES}

1. Priori SG, Wilde AA, Horie M, Cho Y, Behr ER, Berul C, et al. HRS/EHRA/APHRS expert consensus statement on the diagnosis and management of patients with inherited primary arrhythmia syndromes: document endorsed by HRS, EHRA, and APHRS in May 2013 and by ACCF, AHA, PACES, and AEPC in June 2013. Europace. 2013 Oct;15(10):1389-406.

2. Priori SG, Blomstrom-Lundqvist C, Mazzanti A, Blom N, Borggrefe M, Camm J et al. 2015 ESC guidelines for the management of patients with ventricular arrhythmias and the prevention of sudden cardiac death. Europace 2015;17: 1601-87.

3. Hocini M, Pison L, Proclemer A, Larsen TB, Madrid A, Carina Blomström-Lundqvist C. Diagnosis and management of patients with inherited arrhythmia syndromes in Europe: results of the European Heart Rhythm Association Survey. Europace. 2014;16(4):600-603.

4. Nademanee K, Hocini M, Haïssaguerre M. Epicardial substrate ablation for Brugada syndrome. Heart Rhythm. 2017;14(3):457-461.

5. Pappone C, Brugada J, Vicedomini G, Ciconte G, Manguso F, Saviano M et al. Electrical Substrate Elimination in 135 Consecutive Patients With Brugada Syndrome. Circ Arrhythm Electrophysiol. 2017;10(5):e005053.

6. Conte G, Dewals W, Sieira J, de Asmundis C, Ciconte G, Chierchia GB et al. Druginduced Brugada syndrome in children: clinical features, device-based management, and long-term follow-up. J Am Coll Cardiol. 2014;63(21):2272-2279.

7. Pappone C, Radinovic A, Manguso F, Vicedomini G, Sala, Sacco FM et al. New-onset atrial fibrillation as first clinical manifestation of latent Brugada Syndrome: prevalence and clinical significance. Eur Heart J. 2009;30(24):2985-2992. 
8. Hasdemir C, Payzin S, Kocabas U, Sahin H, Yildirim N, Alpet A et al. High prevalence of concealed Brugada syndrome in patients with atrioventricular nodal reentrant tachycardia. Heart Rhythm. 2015;12(7):1584-1594.

9. Tester DJ, Medeiros-Domingo A, Will ML, Ackerman MJ. Unexplained drownings and the cardiac cha nnelopathies: a molecular autopsy series. Mayo Clin Proc. 2011 Oct;86(10):9417.

10. Lahrouchi N, Raju H, Lodder EM, et al. Utility of Post-Mortem Genetic Testing in Cases of Sudden Arrhythmic Death Syndrome. J Am Coll Cardiol. 2017;69(17):2134-2145.

11. Wong LC, Roses-Noguer F, Till JA, Behr ER. Cardiac evaluation of pediatric relatives in sudden arrhythmic death syndrome: a 2-center experience. Circ Arrhythm Electrophysiol. 2014;7(5):800-806.

12. Conte G, de Asmundis C, Ciconte G, Julia J, Sieira J, Chierchia GB et al. Follow-up from childhood to adulthood of individuals with family history of Brugada Syndrome and normal electrocardiograms. JAMA. 2014;312(19):2039-2041.

13. Sroubek J, Probst V, Mazzanti A, Delise P , Hevia JC, Ohkubo K et al.. Programmed Ventricular Stimulation for Risk Stratification in the Brugada Syndrome: A Pooled Analysis. Circulation. 2016;133(7):622-630.

14. Wilde AA, Nademanee K. Epicardial Substrate Ablation in Brugada Syndrome: Time for a Randomized Trial!. Circ Arrhythm Electrophysiol. 2015;8(6):1306-1308. 
Figure 1. Presence of IADs units in the EHRA Research network and ECGen members centres.

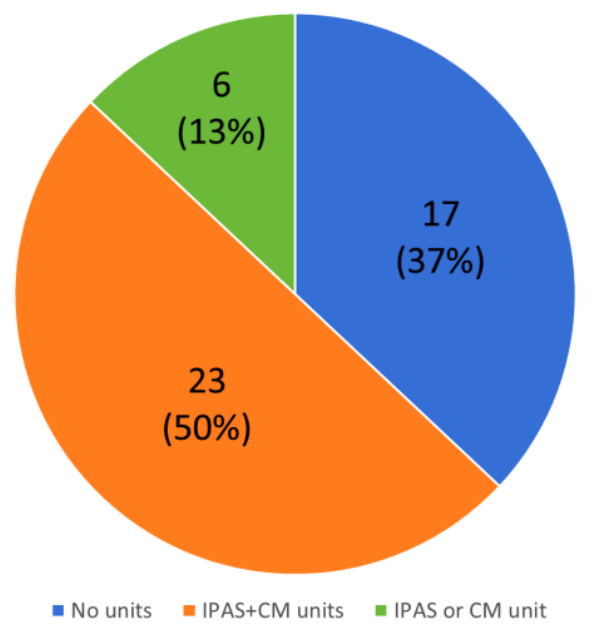

Total answers: 46

IPAS inherited primary arrhythmia syndromes; CM cardiomyopathies

Figure 2. Indications for pharmacological challenge.

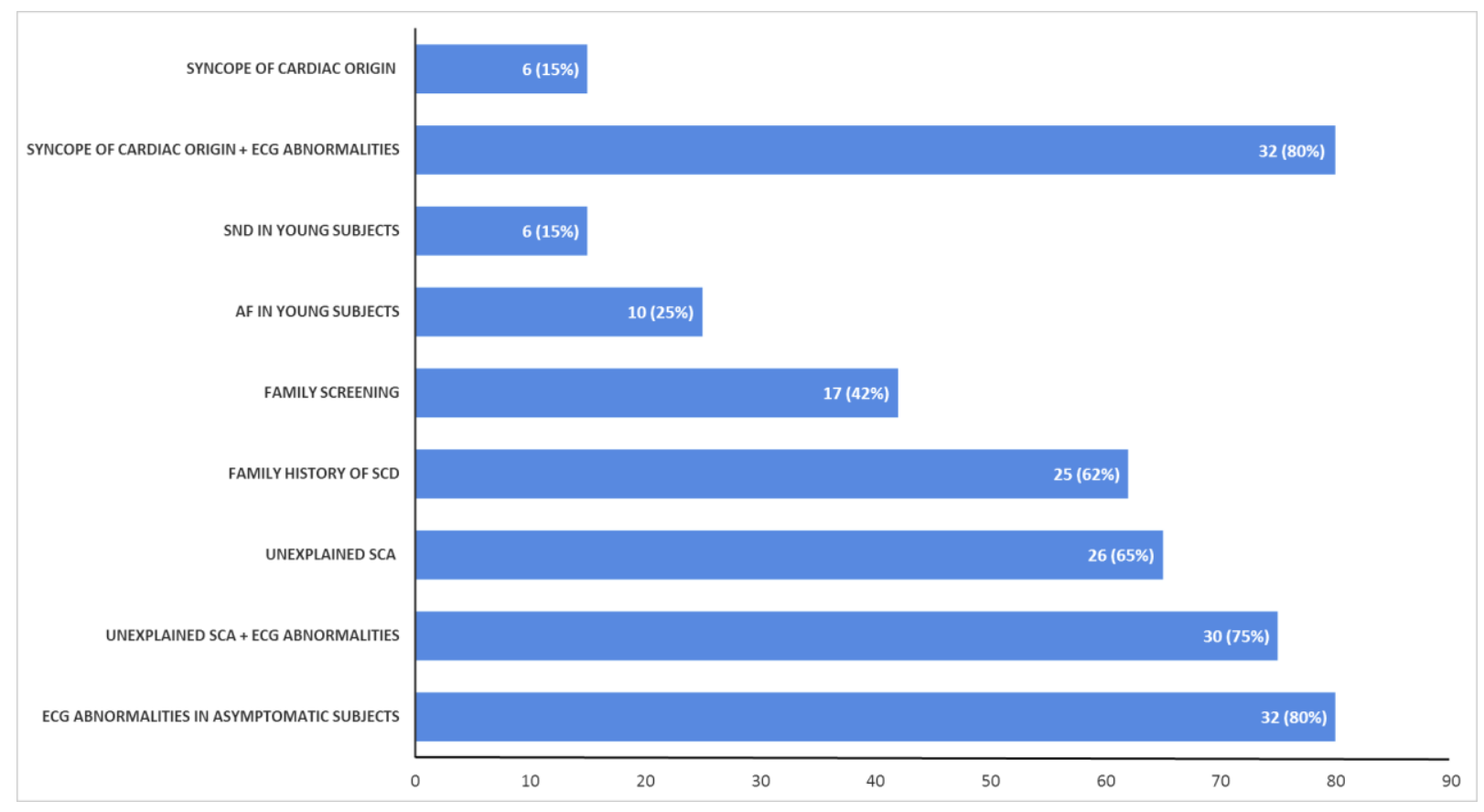

Total answers: 40

SND sinus node dysfunction; AF atrial fibrillation; SCD sudden cardiac death; SCA sudden cardiac arrest 
Figure 3. Rate of diagnostic exams in patients with IADs.

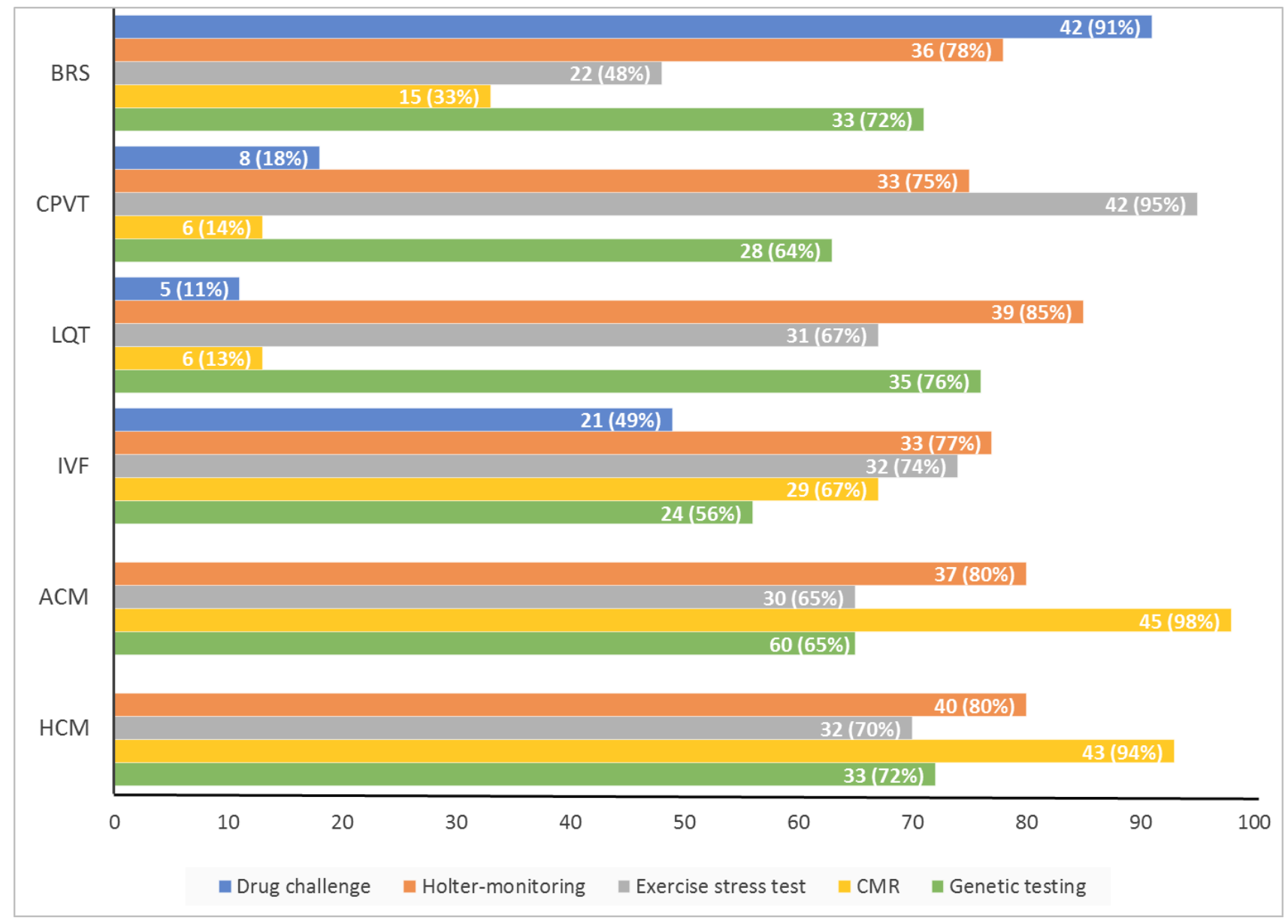

Total answers: BrS 46; CPVT 44; LQT 46; IVF 43; ACM 44; HCM 46.

BrS Brugada syndrome; CPVT Catecholaminergic polymorphic ventricular tachycardia; LQT long-QT syndrome; IVF idiopathic ventricular fibrillation; ACM Arrhythmogenic cardiomyopathy; HCM hypertrophic cardiomyopathy; CMR cardiac magnetic resonance. 
Figure 4. Rate of genetic testing in centres with and without dedicated IAS units.

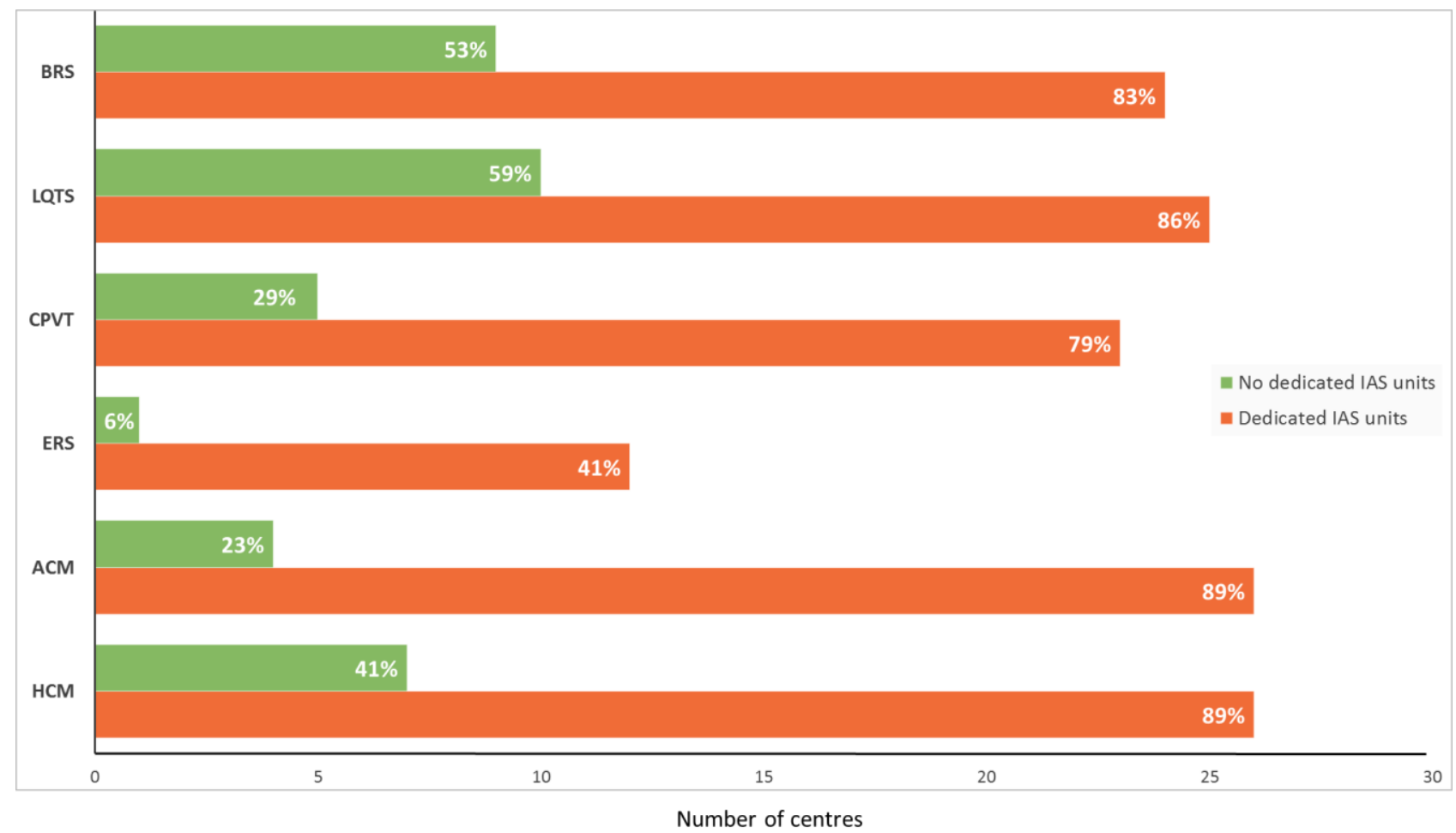

Total answers: 46 (29 centres with dedicated IAS units and 17 centres with no dedicated IAS units)

BrS Brugada syndrome; CPVT Catecholaminergic polymorphic ventricular tachycardia; LQTS long-QT syndrome; IVF idiopathic ventricular fibrillation; ACM Arrhythmogenic cardiomyopathy; HCM hypertrophic cardiomyopathy

Figure 5. Rate of specific indications to PVS in BrS.

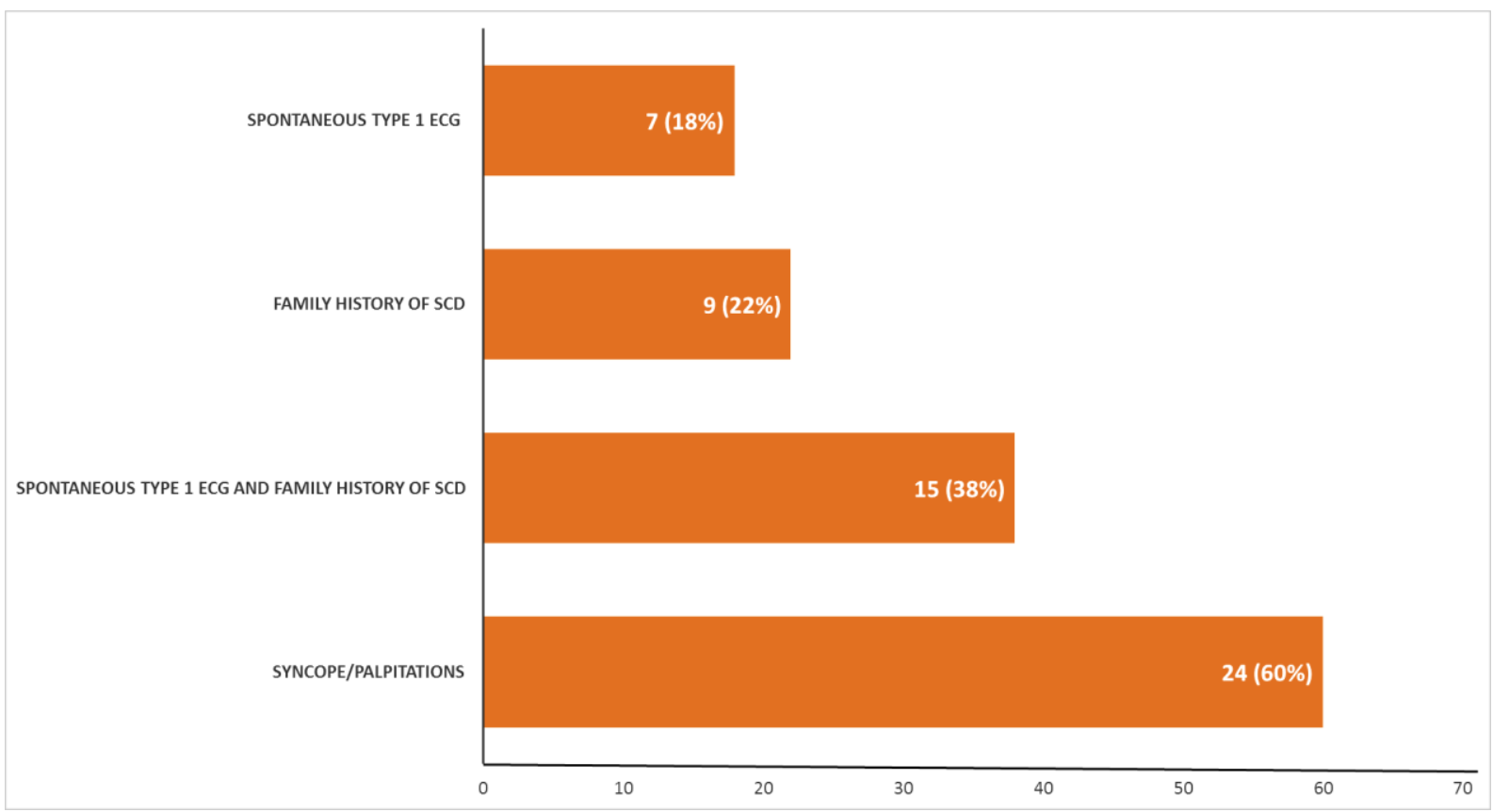

Total answers: 40

SCD sudden cardiac death. 
Figure 6. Drug challenge in pediatric BrS family members.

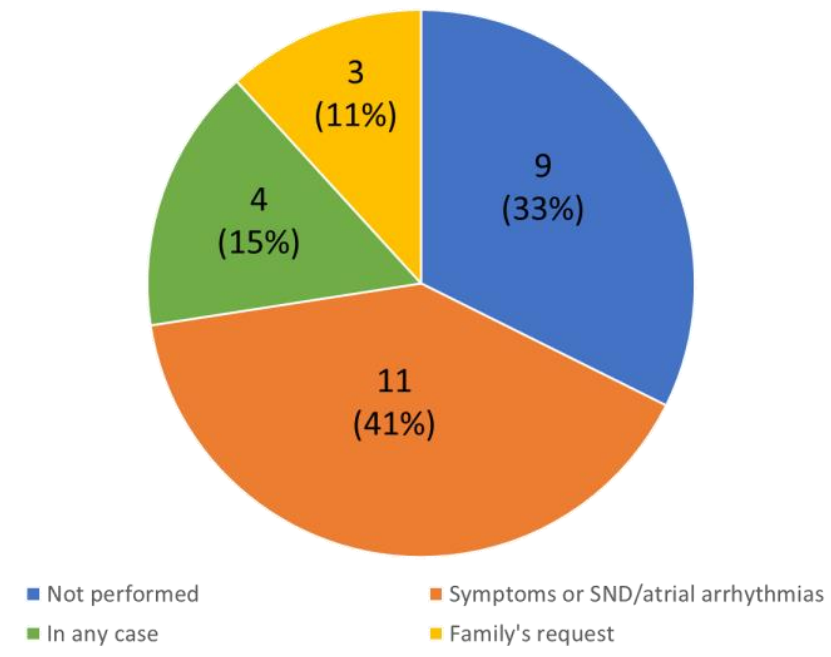

Total answers: 27

SND sinus node dysfunction. 


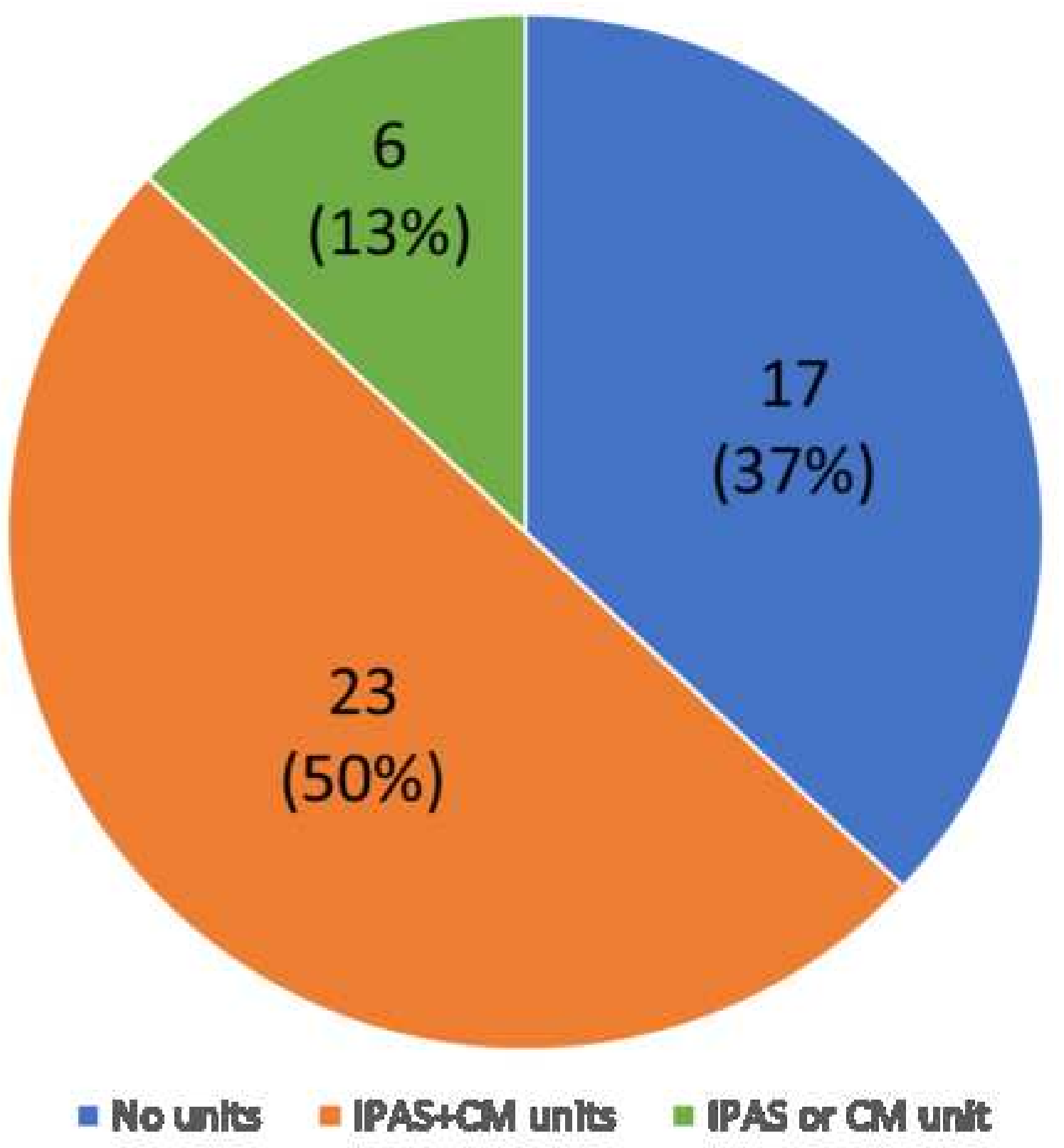




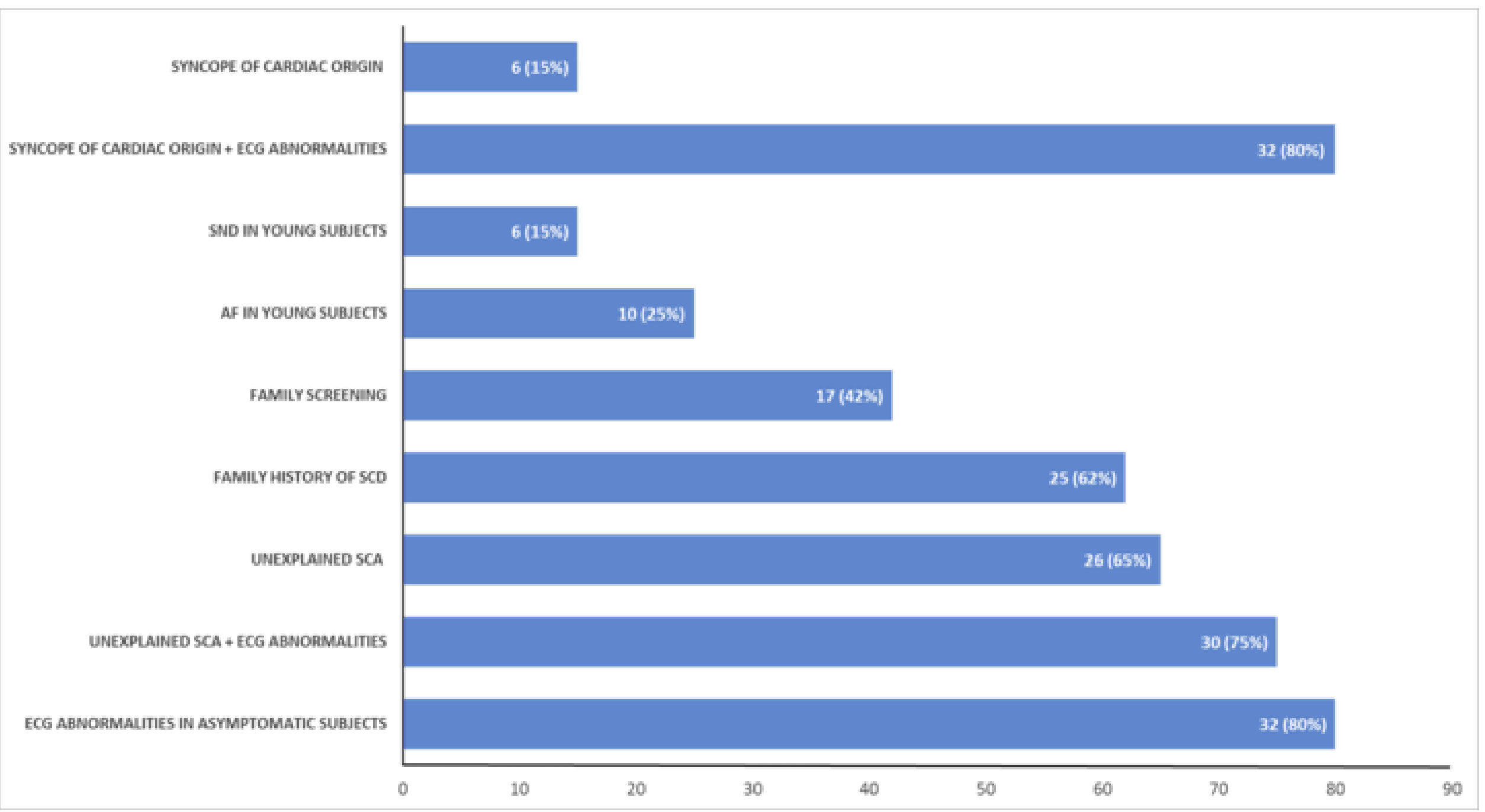




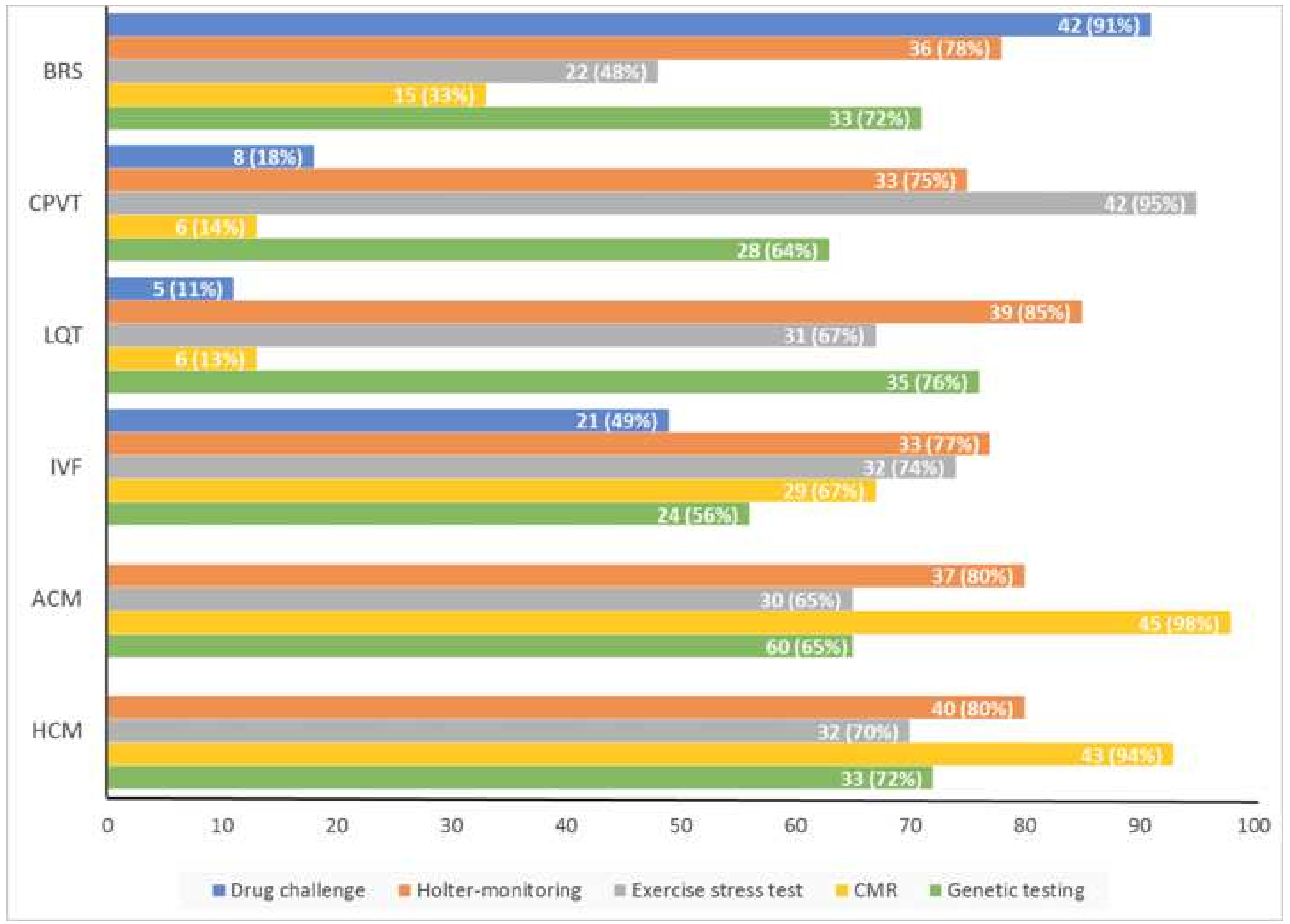




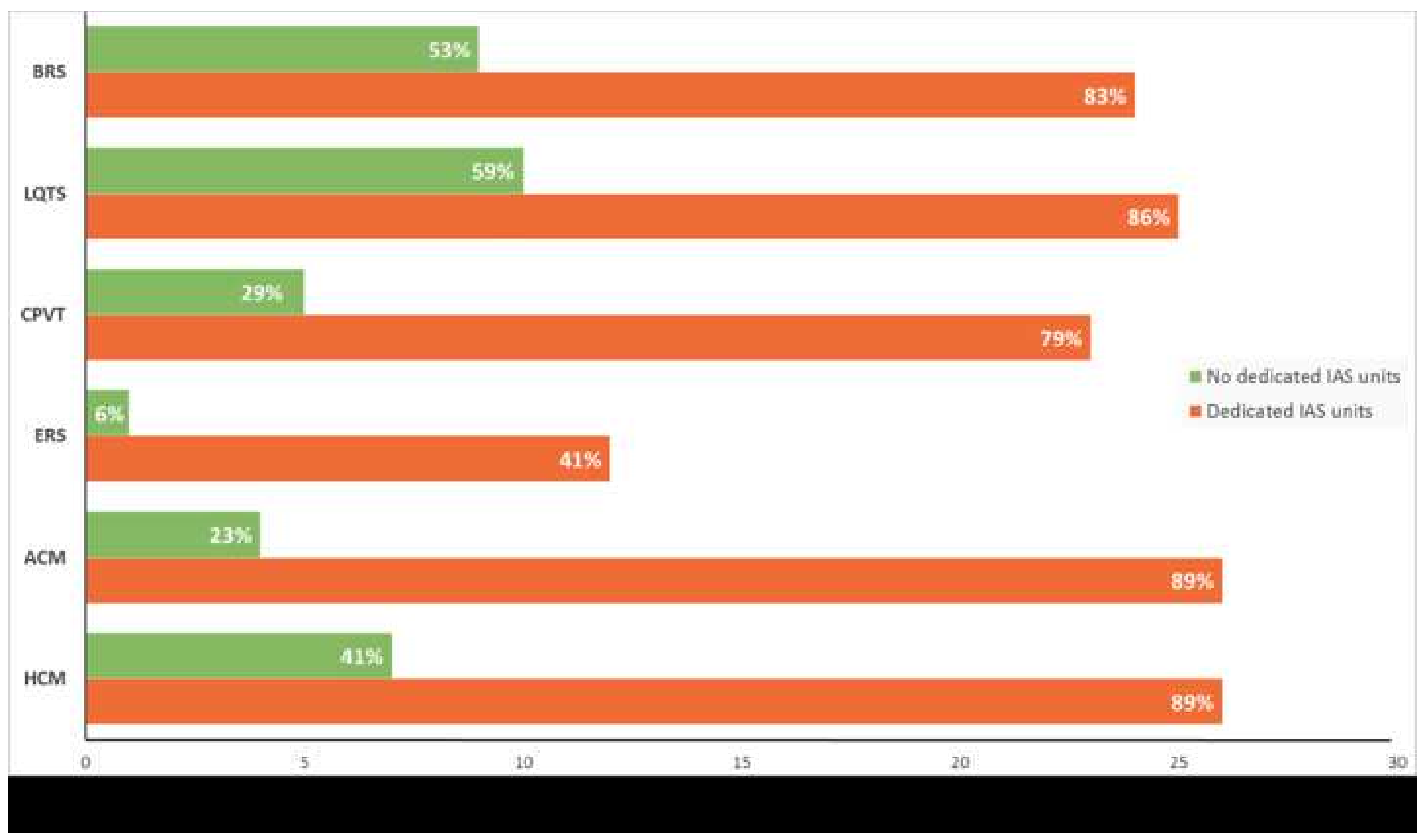




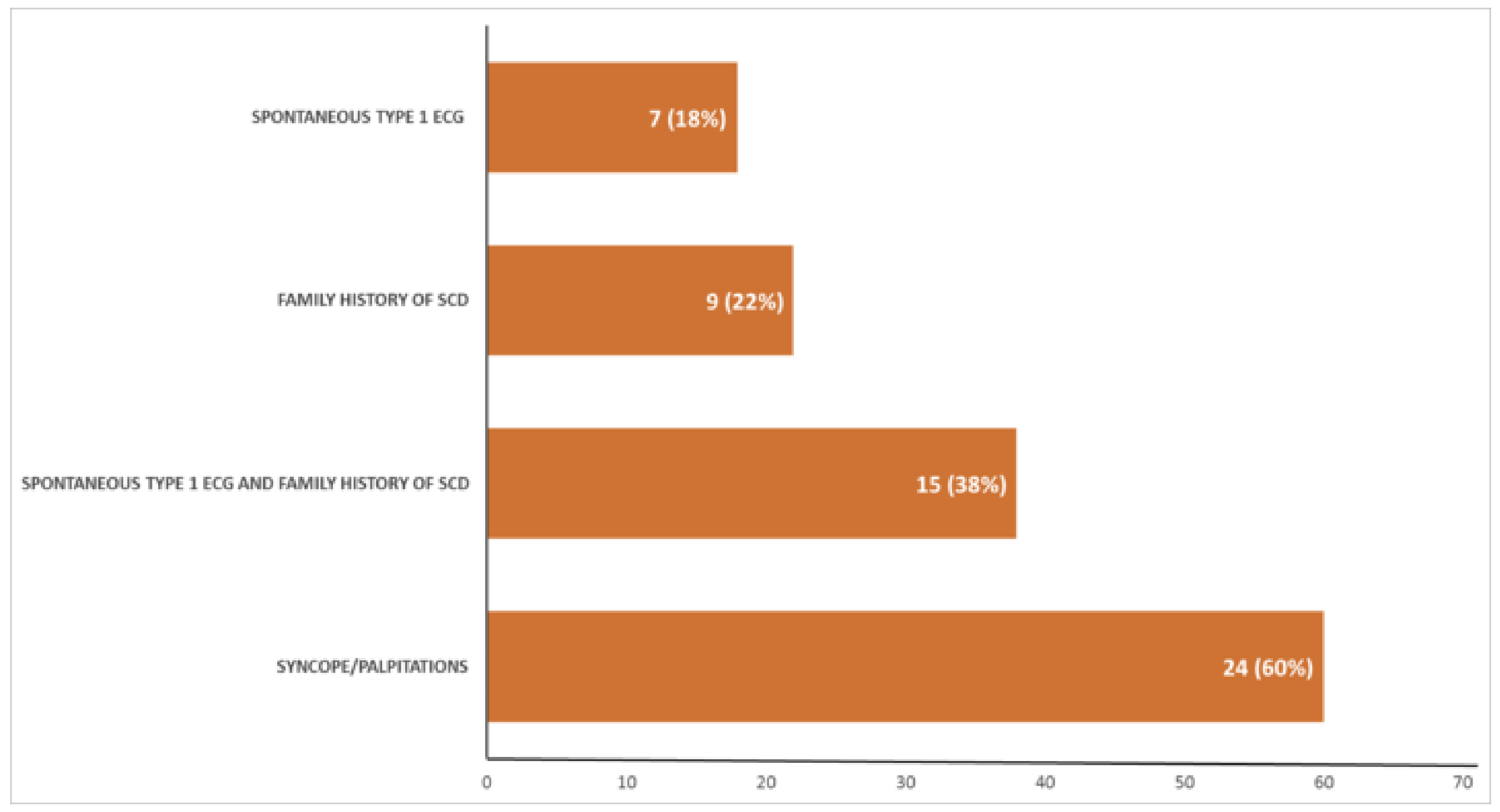




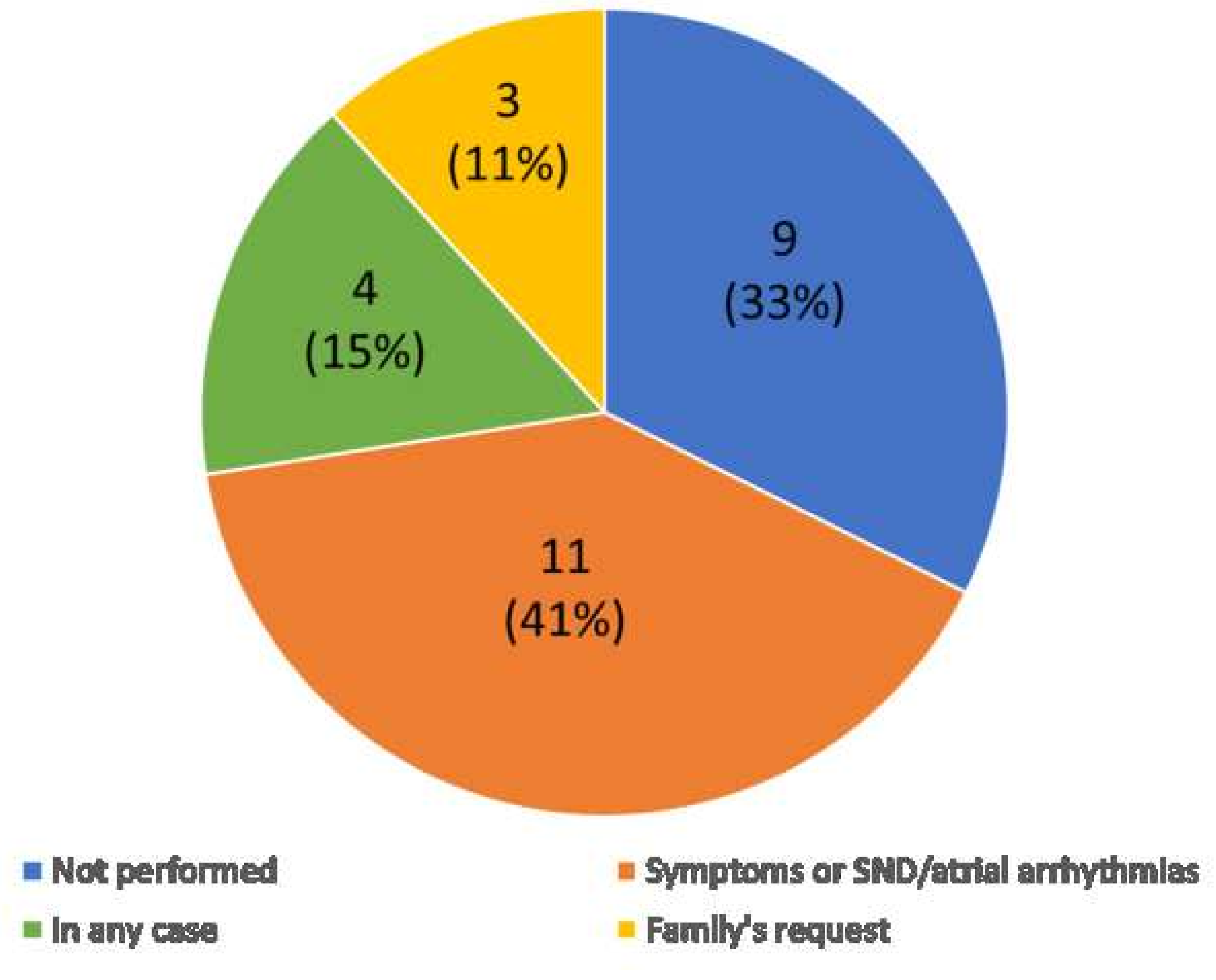

- Not performed

= In any case
= Symptoms or SMD/atrial anryythmlas

= Famlly's request 